\title{
Results on production and decay of B hadrons and onia and $X(5568)$ state search in CMS
}

\section{Sergey Polikarpov on behalf of the CMS Collaboration*}

National Research Nuclear University “Moscow Engineering Physics Institute” (MEPhI),

Moscow, Russia

E-mail: sergey.polikarpov@cern.ch

We present precise measurements of B hadron lifetimes performed by the CMS experiment at the LHC in proton-proton collisions at $\sqrt{s}=8 \mathrm{TeV}$. The first observation of $\Upsilon$ pair production and the search for $\mathrm{X}(5568)$ state are reported. The analyses are performed using a data sample corresponding to an integrated luminosity of about $20 \mathrm{fb}^{-1}$.

The European Physical Society Conference on High Energy Physics

5-12 July, 2017

Venice

${ }^{*}$ Speaker. 
The CMS experiment [1] at the LHC has a wide program in the Heavy Flavor physics sector. In this work, the following recent CMS results on B hadron and quarkonium production and properties are reported:

- Precise measurements of $\mathrm{B}^{0}, \mathrm{~B}_{\mathrm{s}}^{0}, \mathrm{~B}_{\mathrm{c}}^{+}$and $\Lambda_{\mathrm{b}}^{0}$ lifetimes in the following decay channels: $\mathrm{B}^{0} \rightarrow \mathrm{J} / \psi \mathrm{K}^{* 0}\left(\mathrm{~K}^{* 0} \rightarrow \mathrm{K}^{+} \pi^{-}\right), \mathrm{B}^{0} \rightarrow \mathrm{J} / \psi \mathrm{K}_{S}^{0}\left(\mathrm{~K}_{S}^{0} \rightarrow \pi^{+} \pi^{-}\right), \mathrm{B}_{\mathrm{s}}^{0} \rightarrow \mathrm{J} / \psi \phi\left(\phi \rightarrow \mathrm{K}^{+} \mathrm{K}^{-}\right), \mathrm{B}_{\mathrm{s}}^{0} \rightarrow$ $\mathrm{J} / \psi \pi^{+} \pi^{-}, \mathrm{B}_{\mathrm{c}}^{+} \rightarrow \mathrm{J} / \psi \pi^{+}$and $\Lambda_{\mathrm{b}}^{0} \rightarrow \mathrm{J} / \psi \Lambda^{0}\left(\Lambda^{0} \rightarrow \mathrm{p} \pi^{-}\right)$. In every case, $\mathrm{J} / \psi \rightarrow \mu^{+} \mu^{-}$decay is used.

- First observation of double $\Upsilon(1 \mathrm{~S})$ production in proton-proton collisions and measurement of its cross section in the rapidity range $|y(\Upsilon)|<2.0$.

- Search for $\mathrm{X}(5568)$ state decaying into $\mathrm{B}_{\mathrm{s}}^{0} \pi^{ \pm}$, with negative result and upper limit set on the production of $\mathrm{X}(5568)$.

\section{Precise measurements of $B$ hadron lifetimes}

Models inspired by Quantum Chromodynamics (QCD) provide accurate predictions of lifetimes of hadrons containing a b-quark. Precise experimental measurements are important to test those predictions.

B hadron decays are reconstructed through the decay $\mathrm{J} / \psi \rightarrow \mu^{+} \mu^{-}$, which is also used to trigger the event readout. The $\mathrm{J} / \psi$ candidate is combined with a track (for $\mathrm{B}_{\mathrm{c}}^{+} \rightarrow \mathrm{J} / \psi \pi^{+}$case) or with two oppositely-charged tracks (for $\mathrm{B}^{0} \rightarrow \mathrm{J} / \psi \mathrm{K}^{* 0}, \mathrm{~B}_{\mathrm{s}}^{0} \rightarrow \mathrm{J} / \psi \pi^{+} \pi^{-}$and $\mathrm{B}_{\mathrm{s}}^{0} \rightarrow \mathrm{J} / \psi \phi$ ) to form a vertex, which is required to be significantly displaced from the primary vertex (PV). For $\Lambda_{\mathrm{b}}^{0} \rightarrow \mathrm{J} / \psi \Lambda^{0}$ and $\mathrm{B}^{0} \rightarrow \mathrm{J} / \psi \mathrm{K}_{S}^{0}$ decays, the long-lived hadrons are reconstructed from two-prong displaced vertices (in case of $\Lambda^{0} \rightarrow \mathrm{p} \pi^{-}$, the track with higher momentum of the two is selected as a proton candidate). Thresholds on the track transverse momentum, track and vertex fits quality and masses of intermediate states are applied to reduce the background.

The lifetimes are extracted with a fit to the 3-dimensional distribution of the reconstructed invariant mass of B hadron candidate, $c t$ and per-event $c t$ uncertainty. Here $t$ denotes the measured proper decay time of a $\mathrm{B}$ hadron candidate, calculated from the transverse decay length $L_{x y}$ and $p_{\mathrm{T}}$ of the candidate and the world-average mass of $\mathrm{B}$ hadron $M$ as

$$
t=L_{x y} \frac{M}{p_{\mathrm{T}}} .
$$

The fit model consists of the signal model, background model and, in case of the $\mathrm{B}_{\mathrm{s}}^{0} \rightarrow \mathrm{J} / \psi \pi^{+} \pi^{-}$ channel, the contribution from other partially reconstructed and other misidentified $\mathrm{B}$ hadron decays. The reconstruction efficiency depends on $c t$ (an example is shown in Fig. 1 for $\mathrm{B}^{0} \rightarrow \mathrm{J} / \psi \mathrm{K}^{* 0}$ decay), and this effect is taken into account in the fits.

For the $\mathrm{B}_{\mathrm{c}}^{+}$lifetime, a different method is used to reduce systematic uncertainties. The yields of $\mathrm{B}_{\mathrm{c}}^{+} \rightarrow \mathrm{J} / \psi \pi^{+}$and $\mathrm{B}^{+} \rightarrow \mathrm{J} / \psi \mathrm{K}^{+}$decays are obtained in the intervals of $c t$ and their ratio is fit with an exponential function, as shown in Fig. 2. From the result of the fit, the $\mathrm{B}_{\mathrm{c}}^{+}$lifetime is obtained using the known lifetime of the $\mathrm{B}^{+}$meson. The same method was used by LHCb collaboration to measure the $\mathrm{B}_{\mathrm{c}}^{+}$lifetime in the same decay mode [3]. 


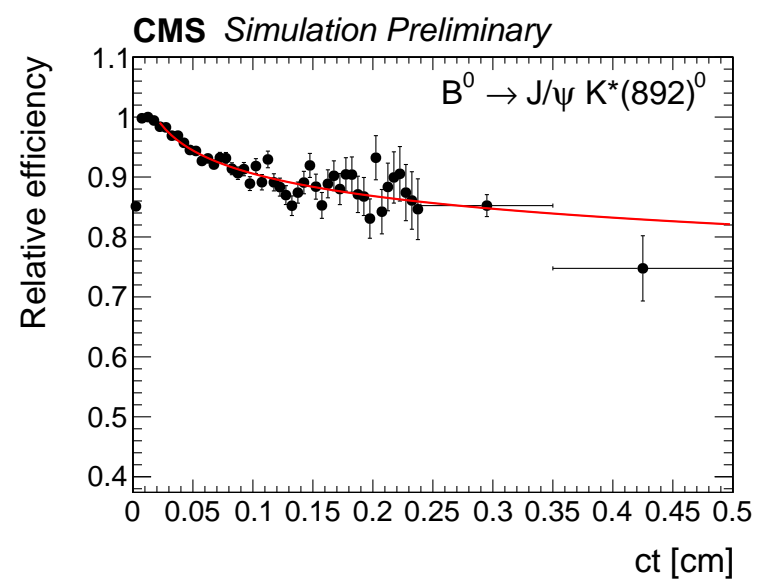

Figure 1: Efficiency dependence on $c t$ for $\mathrm{B}^{0} \rightarrow \mathrm{J} / \psi \mathrm{K}^{* 0}$ [2] fitted with inverse power function (efficiency scale is arbitrary).

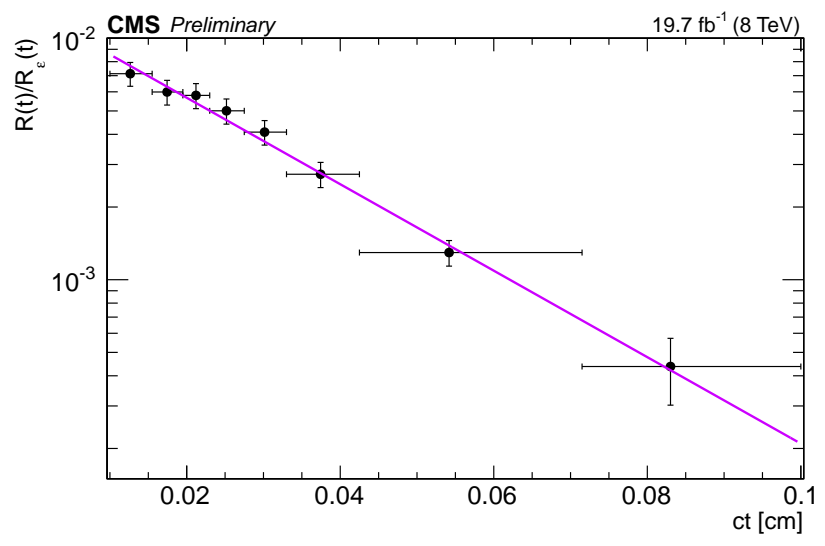

Figure 2: Ratio of efficiency-corrected $c t$ distributions for $\mathrm{B}_{\mathrm{c}}^{+}$and $\mathrm{B}^{+}$signals [2]. The line shows the fit result with an exponential function.

Main sources of systematic uncertainties come from the finite size of the simulation sample, algorithm of PV selection and possible detector misalignment.

The measured lifetimes are [2]:

$$
\begin{aligned}
& c \tau_{\mathrm{B}^{0}}=453.0 \pm 1.6 \text { (stat) } \pm 1.5 \text { (syst) } \mu \mathrm{m}\left(\text { in } \mathrm{J} / \psi \mathrm{K}^{* 0}\right), \\
& c \tau_{\mathrm{B}^{0}}=457.8 \pm 2.7 \text { (stat) } \pm 2.7 \text { (syst) } \mu \mathrm{m}\left(\text { in } \mathrm{J} / \psi \mathrm{K}_{S}^{0}\right), \\
& c \tau_{\mathrm{B}_{\mathrm{s}}^{0}}=502.7 \pm 10.2 \text { (stat) } \pm 3.2 \text { (syst) } \mu \mathrm{m}\left(\text { in } \mathrm{J} / \psi \pi^{+} \pi^{-}\right), \\
& c \tau_{\mathrm{B}_{\mathrm{s}}^{0}}=443.9 \pm 2.0 \text { (stat) } \pm 1.1 \text { (syst) } \mu \mathrm{m}(\text { in } \mathrm{J} / \psi \phi), \\
& c \tau_{\Lambda_{\mathrm{b}}^{0}}=442.9 \pm 8.2 \text { (stat) } \pm 2.7 \text { (syst) } \mu \mathrm{m}, \\
& c \tau_{\mathrm{B}_{\mathrm{c}}^{+}}=162.3 \pm 8.2 \text { (stat) } \pm 4.7 \text { (syst) } \pm 0.1\left(\tau_{\mathrm{B}^{+}}\right) \mu \mathrm{m},
\end{aligned}
$$

where the first uncertainty is statistical and the second is systematic. All results are in agreement 
with the current world average values and the accuracy for each channel is at the level of or better than the previous results in the respective channel.

\section{Observation of $\Upsilon(1 S)$ pair production}

Quarkonium pair production cross section measurements are one of the key measurements in understanding Single Parton Scattering (SPS) and Double Parton Scattering (DPS) contributions. We report observation of simultaneous production of two $\Upsilon(1 \mathrm{~S})$ mesons in pp collisions at $\sqrt{s}=$ $8 \mathrm{TeV}$. The dimuon decay is used to reconstruct $\Upsilon$ candidates. Four muons are required to have a common vertex and the rapidity of the reconstructed $\Upsilon$ candidates is required to be $|y(\Upsilon)|<2.0$.

Two-dimensional unbinned fit to the invariant mass of reconstructed $M_{\mu^{+} \mu^{-}}^{1}$ and $M_{\mu^{+} \mu^{-}}^{2}$ combinations is used, where $M_{\mu^{+} \mu^{-}}^{1}>M_{\mu^{+} \mu^{-}}^{2}$. If an event contains multiple $\Upsilon \Upsilon$ candidates, it is discarded. No signs of $\Upsilon(3 S)$ and higher states are observed, therefore, their contributions are neglected. The fit includes pure combinatorial background component, $\Upsilon(1 S) \Upsilon(1 S)$ and $\Upsilon(2 S) \Upsilon(1 S)$ signal components and the contributions from $\Upsilon(1 \mathrm{~S})$ or $\Upsilon(2 \mathrm{~S})$ and two unassociated muons. The fit result is presented in Fig. 3 and returns the number of $\Upsilon(1 \mathrm{~S}) \Upsilon(1 \mathrm{~S})$ signal candidates $N_{\text {sig } 1}=38 \pm 7$ with a corresponding significance of $9.6 \sigma$ (including systematic uncertainties) [4].
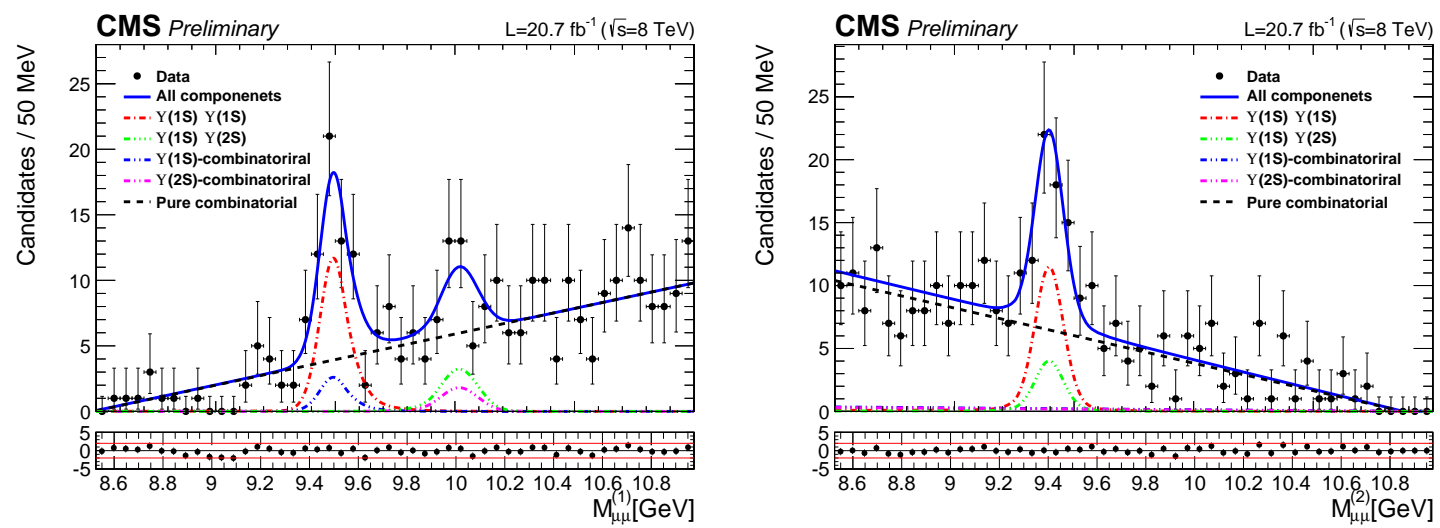

Figure 3: Projections of the 2D fit. Left: invariant mass distribution of heavier dimuon $\left(M_{\mu^{+} \mu^{-}}^{1}\right)$ and right: invariant mass distribution of lighter dimuon $M_{\mu^{+} \mu^{-}}^{1}$ [4].

The cross section $\sigma_{\mathrm{T}}$ of $\Upsilon(1 \mathrm{~S})$ pair production is measured to be $\sigma_{\mathrm{T}}=68.8 \pm 12.7$ (stat) \pm 7.4 (syst) \pm 2.8 (BR) pb [4] in the region $|y(\Upsilon)|<2.0, p_{\mathrm{T}}(\Upsilon(1 \mathrm{~S}))<50 \mathrm{GeV}$, where the uncertainties are statistical, systematic and from the branching fraction knowledge, respectively, and isotropic decay of $\Upsilon(1 \mathrm{~S})$ is assumed. Cross section ranges from $-38 \%$ to $+36 \%$ from the value above when different $\Upsilon(1 \mathrm{~S})$ polarizations are assumed.

\section{Search for $\mathrm{X}(\mathbf{5 5 6 8})$}

The claim for the $\mathrm{X}(5568)$ state decaying into $\mathrm{B}_{\mathrm{s}}^{0} \pi^{ \pm}$by the D0 Collaboration [5] raised many controversial discussions in the exotic hadron community. The new state was reported to have a mass of $5567.8 \pm 2.9(\text { stat })_{-1.9}^{+0.9}$ (syst) $\mathrm{MeV}$ and natural width $21.9 \pm 6.4(\text { stat })_{-2.5}^{+5.0}$ (syst) MeV. 
The relative production of $\mathrm{X}(5568)$ w.r.t. $\mathrm{B}_{\mathrm{s}}^{0}$ meson, multiplied by $\mathscr{B}\left(\mathrm{X}(5568) \rightarrow \mathrm{B}_{\mathrm{s}}^{0} \pi^{ \pm}\right)$, was measured to be $\rho_{\mathrm{X}}=(8.6 \pm 1.9 \pm 1.4) \%$ for $p_{\mathrm{T}}\left(\mathrm{B}_{\mathrm{s}}^{0}\right)>10 \mathrm{GeV}$ in proton-antiproton collisions at $\sqrt{s}=1.96 \mathrm{TeV}$ [5]. The LHCb Collaboration reported a negative result of the $\mathrm{X}(5568)$ search and set an upper limit on $\rho_{\mathrm{X}}: \rho_{\mathrm{X}}<2.4 \% @ 95 \% \mathrm{CL}$ for $p_{\mathrm{T}}\left(\mathrm{B}_{\mathrm{s}}^{0}\right)>10 \mathrm{GeV}[6]$.

We report a negative search result for $\mathrm{X}(5568)$ at the $\mathrm{CMS}$ experiment. The $\mathrm{B}_{\mathrm{s}}^{0}$ candidates are reconstructed using the decay channel $\mathrm{B}_{\mathrm{s}}^{0} \rightarrow \mathrm{J} / \psi \phi$, where $\mathrm{J} / \psi \rightarrow \mu^{+} \mu^{-}$and $\phi \rightarrow \mathrm{K}^{+} \mathrm{K}^{-}$decays are used. The same trigger used in the $\mathrm{B}$ hadrons lifetime measurement (Sect. 1) is used in this analysis. The selection requirements are similar to those used in the measurement of the CP-violating phase $\phi_{\mathrm{S}}$ in the same decay [7].

The fit to the reconstructed $\mathrm{J} / \psi \phi$ invariant mass distribution returns about $50,000 \mathrm{~B}_{\mathrm{s}}^{0}$ signal candidates [8]. The pions coming from the $\mathrm{B}_{\mathrm{s}}^{0}$ production $\mathrm{PV}$ and satisfying $p_{\mathrm{T}}>0.5 \mathrm{GeV}$ requirement are combined with $\mathrm{B}_{\mathrm{s}}^{0}$ candidates to form $\mathrm{B}_{\mathrm{s}}^{0} \pi^{ \pm}$candidates. The obtained $M\left(\mathrm{~B}_{\mathrm{s}}^{0} \pi^{ \pm}\right)$invariant mass distribution is shown in Fig. 4 (a), compared between the $\mathrm{B}_{\mathrm{s}}^{0}$ signal region and the $\mathrm{B}_{\mathrm{s}}^{0}$ sideband regions. No significant structures are visible near $5568 \mathrm{MeV}$ nor in the whole mass region $5.5-5.9 \mathrm{GeV}$, and the distribution from the $\mathrm{B}_{\mathrm{s}}^{0}$ signal region is found to be close to that of $\mathrm{B}_{\mathrm{s}}^{0}$ sidebands. The fit to the distribution in the $\mathrm{B}_{\mathrm{s}}^{0}$ signal region with smooth background function and a signal component (Relativistic Breit-Wigner with mass and width fixed to the values measured by the D0, convolved with resolution) is presented in Fig. 4 (b) and returns number of X(5568) signal candidates consistent with zero: $N_{\mathrm{X}}=-175 \pm 134$ [8]. Fits are also performed after different variations of selection criteria, in particular, different requirements on $p_{\mathrm{T}}$ of $\mathrm{B}_{\mathrm{s}}^{0}, \pi^{ \pm}, \mathrm{B}_{\mathrm{s}}^{0} \pi^{ \pm}$candidates or reconstruction quality criteria. In each case, the signal yield is found to be consistent with zero.
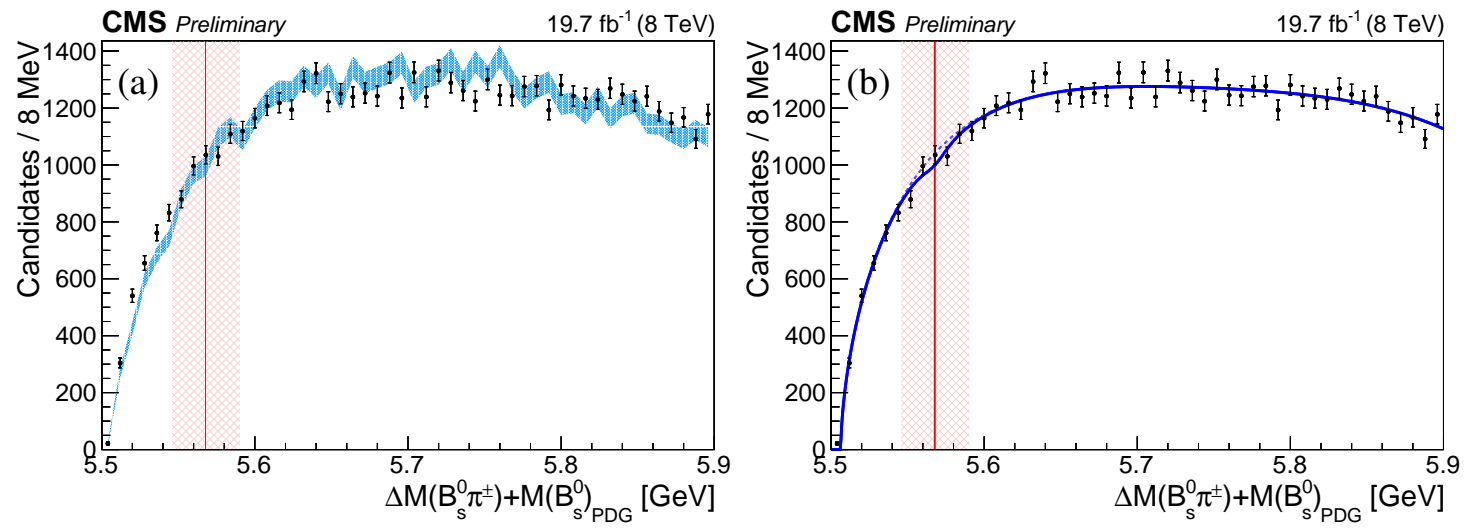

Figure 4: (a): $\mathrm{B}_{\mathrm{s}}^{0} \pi^{ \pm}$invariant mass distribution for the $\mathrm{B}_{\mathrm{s}}^{0}$ signal region and $\mathrm{B}_{\mathrm{s}}^{0}$ sidebands. (b): fit to the $\mathrm{B}_{\mathrm{s}}^{0} \pi^{ \pm}$invariant mass distribution in the $\mathrm{B}_{\mathrm{s}}^{0}$ signal region [8].

The reconstruction procedure is verified by removing the requirement on $\mathrm{K}^{+} \mathrm{K}^{-}$mass, allowing the $\mathrm{B}^{0} \rightarrow \mathrm{J} / \psi \mathrm{K}^{+} \pi^{-}$decay to contribute to the reconstructed $\mathrm{B}_{\mathrm{s}}^{0} \rightarrow \mathrm{J} / \psi \mathrm{K}^{+} \mathrm{K}^{-}$sample. Consequently, the decays of excited $\mathrm{B}$ meson states $\mathrm{B}_{1,2}^{(*)+} \rightarrow \mathrm{B}^{(*) 0} \pi^{+}$produce clear excess in the reconstructed $\mathrm{B}_{\mathrm{s}}^{0} \pi^{ \pm}$invariant mass distribution [8].

An upper limit on $\rho_{\mathrm{X}}$ is set: $\rho_{\mathrm{X}}<3.9 \% @ 95 \% \mathrm{CL}$ for $p_{\mathrm{T}}\left(\mathrm{B}_{\mathrm{s}}^{0}\right)>10 \mathrm{GeV}$ [8]. The main source of systematic uncertainty is the knowledge of the relative efficiency $\varepsilon_{\mathrm{X}(5568)} / \varepsilon_{\mathrm{B}_{\mathrm{s}}^{0}}$ from simulation. 


\section{Summary and acknowledgments}

In summary, CMS continues contributing to the Heavy Flavour physics sector. The measured lifetimes of $B$ hadrons have precision at the level of or better than the previous experiments. $\Upsilon(1 S)$ pair production in proton proton collisions is observed for the first time and its cross section is measured. The $\mathrm{X}(5568)$ state claimed by the $\mathrm{D} 0$ collaboration is searched for, and a negative result and an upper limit are reported, in agreement with LHCb collaboration.

The author's work was supported by the Russian Ministry of Education and Science contracts 3.2989.2017 and 14.A12.31.0006. The author would like to thank the CMS Collaboration for the content of the presentation and the EPS 2017 conference organizers for the excellent program and opportunity to present these results.

\section{References}

[1] The CMS Collaboration, The CMS experiment at the CERN LHC, JINST 3 (2008) S08004.

[2] The CMS Collaboration, Precision lifetime measurements of $\mathrm{b}$ hadrons reconstructed in final states with a $\mathrm{J} / \psi$ meson, CMS-PAS-BPH-13-008.

[3] LHCb Collaboration, Measurement of the lifetime of the $\mathrm{B}_{\mathrm{c}}^{+}$meson using the $\mathrm{B}_{\mathrm{c}}^{+} \rightarrow \mathrm{J} / \psi \pi^{+}$decay mode, Phys. Lett. B 742 (2015) 29, [arxiv:1411.6899].

[4] The CMS Collaboration, Observation of $Y(1 S)$ pair production in proton-proton collisions at $\sqrt{s}=8 \mathrm{TeV}, \mathrm{JHEP} 05$ (2017) 013, [arXiv:1610.07095].

[5] D0 Collaboration, Evidence for a $B_{s}^{0} \pi^{ \pm}$State, Phys. Rev. Lett. 117 (2016) 022003, [arXiv:1602.07588].

[6] LHCb Collaboration, Search for structure in the $B_{s}^{0} \pi^{ \pm}$invariant mass spectrum State, Phys. Rev. Lett. 117 (2016) 152003, [arXiv:1608.00435].

[7] The CMS Collaboration, Measurement of the CP-violating weak phase $\phi_{\mathrm{s}}$ and the decay width difference $\Delta \Gamma_{\mathrm{s}}$ using the $\mathrm{B}_{\mathrm{s}}^{0} \rightarrow \mathrm{J} / \psi \phi(1020)$ decay channel in pp collisions at $\sqrt{s}=8 \mathrm{TeV}$, Phys. Lett. B 757 (2016) 97, [arXiv:1507.07527].

[8] The CMS Collaboration, Search for the X(5568) state in $\mathrm{B}_{\mathrm{s}}^{0} \pi^{ \pm}$decays, CMS-PAS-BPH-16-002. 